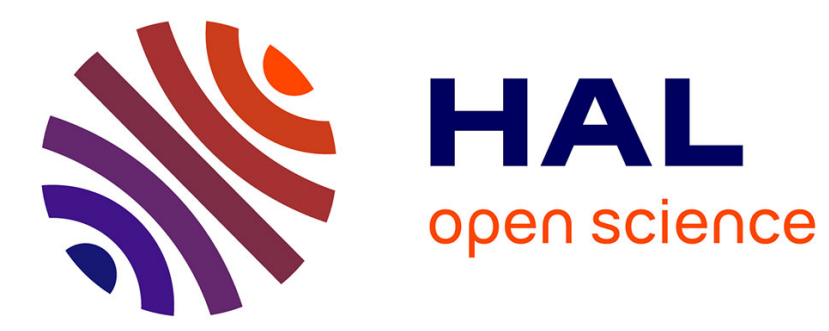

\title{
On Finite-time Stabilization via Relay Feedback Control
} Andrey Polyakov, Laurentiu Hetel

\section{To cite this version:}

Andrey Polyakov, Laurentiu Hetel. On Finite-time Stabilization via Relay Feedback Control. Conference on Decision and Control 2015, IEEE, Dec 2015, Osaka, Japan. hal-01212598

\section{HAL Id: hal-01212598 \\ https://hal.inria.fr/hal-01212598}

Submitted on 6 Oct 2015

HAL is a multi-disciplinary open access archive for the deposit and dissemination of scientific research documents, whether they are published or not. The documents may come from teaching and research institutions in France or abroad, or from public or private research centers.
L'archive ouverte pluridisciplinaire HAL, est destinée au dépôt et à la diffusion de documents scientifiques de niveau recherche, publiés ou non, émanant des établissements d'enseignement et de recherche français ou étrangers, des laboratoires publics ou privés. 


\title{
On Finite-time Stabilization via Relay Feedback Control
}

\author{
Andrey Polyakov and Laurentiu Hetel
}

\begin{abstract}
The problem of finite-time stabilization of multiinput linear system by means of relay feedback is considered. A new control design procedure, which combines convex embedding technique with implicit Lyapunov function method, is developed. The issues of practical implementation of the obtained implicit relay feedback are discussed. Theoretical result is supported by numerical simulation.
\end{abstract}

\section{INTRODUCTION}

Theory of relay automatic control systems has a long outstanding history. Relay feedbacks appeared in the early technological developments of the 19th century. However, the first theoretical study of relay control methods was provided in 1950s [1], [2]. The modern frequency domain approach to analysis of the relay systems can be found in [3]

When the sliding mode control methodology [4] was invented, it suggested to utilize a proper fast relay switching strategy in order to maintain the motion of the control system on a prescribed surface in the state space. Indeed, the classical example of the sliding mode system has the form of relay feedback:

$$
\dot{x}(t)=-\operatorname{sign}[x(t)], t>0, x(0)=x_{0} \in \mathbb{R},
$$

where the sign function is defined as follows: $\operatorname{sign}[\rho]=1$ if $\rho>0$ and $\operatorname{sign}[\rho]=-1$ if $\rho<0$. Any trajectory of this system reaches the state $x=0$ in a finite time and remains thereafter. In fact, finite-time stability frequently accompanies the relay and sliding mode feedback systems [4], [5], [6], [7]. The main application domains of sliding mode approach are electrical and electro-mechanical systems [4], [8].

The modern theoretical framework of hybrid dynamical systems [9], [10] includes the relay feedbacks as a particular case of switched affine systems [11], [12], [13], [14]. Recently, the ideas of convex embedding have been applied in order to design an exponentially stabilizing relay switching law based on the existence of a stabilizing static linear feedback [15]. The present paper addresses the finite-time stabilization of linear multi-input system using relay control. The main goal of the article is to show how the convex embedding procedure can be used with the implicit Lyapunov function method [16], [17], [18] in order to derive a finitetime stabilizing relay feedback.

The paper is organized as follows. The next section presents notations used in the paper. After that, the problem statement and basic assumptions are discussed. Some

This research is supported by ANR ROCC-SYS (ANR-14-CE27-0008). A. Polyakov (andrey.polyakov@inria.fr) is with Non-A team-project, Inria Lille-Nord Europe, 40, avenue Halley, 59650 Villeneuve d'Ascq, France. L. Hetel (laurentiu.hetel@ec-lille.fr) and A. Polyakov are with CRIStAL UMR CNRS 9189, 59650 Villeneuve d'Ascq, France. preliminary facts are considered in Section IV. Next, the main results are presented. Finally, numerical simulation example and concluding remarks are given. Some supporting constructions are provided in Appendix.

\section{NOTATION}

- $\mathbb{R}$ is the set of real numbers; $\mathbb{R}_{+}=\{x \in \mathbb{R}: x>0\}$;

- $\|x\|$ denotes the Euclidian norm of the vector $x \in \mathbb{R}^{n}$;

- range $(B)$ is the column space of the matrix $B \in \mathbb{R}^{n \times m}$;

- $\operatorname{diag}\left\{\lambda_{1}, \ldots, \lambda_{n}\right\}$ is a diagonal matrix with elements $\lambda_{i}$;

- the order relation $P>0(<0, \geq 0, \leq 0)$ for $P \in$ $\mathbb{R}^{n \times n}$ means that $P$ is symmetric and positive (negative) definite (semidefinite);

- if $P>0$ then the matrix $P^{1 / 2}:=B$ is such that $B^{2}=$ $P$

- $\lambda_{\max }(P)$ and $\lambda_{\min }(P)$ denote maximum and minimum eigenvalues of the symmetric matrix $P \in \mathbb{R}^{n \times n}$;

- $I_{m} \in \mathbb{R}^{m \times m}$ is the identity matrix;

- a continuous function $\sigma: \mathbb{R}_{+} \rightarrow \mathbb{R}_{+}$belongs to the class $\mathcal{K}$ if it is monotone increasing and $\sigma(s) \rightarrow+0$ as $s \rightarrow+0$;

- $\operatorname{co}(U)$ is the convex closure of the set $U \subset \mathbb{R}^{n} ; \operatorname{int}\{U\}$ denotes the interior of the set $U$.

\section{Problem Statement}

Let us consider a model of a control system described by the ordinary differential equation (ODE):

$$
\dot{x}(t)=A x(t)+B u(t), \quad t \in \mathbb{R}_{+},
$$

where $x \in \mathbb{R}^{n}$ is the state vector, $u \in \mathbb{R}^{m}$ is the vector of control inputs, $A \in \mathbb{R}^{n \times n}$ is the system matrix, $B \in \mathbb{R}^{n \times m}$ is the matrix of control gains.

It is assumed that the matrices $A$ and $B$ are known, $\operatorname{rank}(B)=m \leq n$ and the pair $(A, B)$ is controllable; the whole state vector $x$ can be measured and utilized for control purpose. The control input $u$ is assumed to be generalized relay, i.e. it can take values from a given discrete set:

$$
u(t) \in \mathcal{U}:=\left\{v_{1}, v_{2}, \ldots, v_{N}\right\}, \quad v_{i} \in \mathbb{R}^{m}, \quad t \in \mathbb{R}_{+},
$$

where $N$ is a natural number. In addition, the assumption

$$
0 \in \operatorname{int}\{\operatorname{co}(\mathcal{U})\} \subset \mathbb{R}^{m}
$$

is possessed in order to guarantee the existence of the stabilizing relay control (see, [15] for the details). As we will see further, this configuration includes as a particular case the classical sliding control generated by $\overline{\text { sign }}$ functions. This control configuration may also be related to the simplex method in [19], [20] and to the stabilization of switched affine systems [12], [13]. Filippov theory of differential 
equations with discontinuous right-hand sides [21] is utilized below in order to take into account the discontinuity of the control law.

The control aim is to stabilize the origin of the system (1) in a finite time and to specify the corresponding set of admissible initial conditions (i.e. the domain of finite-time attraction).

Following the ideas of [15] the relay stabilizing control law can designed in two steps. Initially, some continuous finite-time stabilizing feedback should be selected. For this purpose the method of the Implicit Lyapunov Functions (ILF) is utilized [16], [17], [18], [22]. Next, a proper convex embedding procedure [15] is applied in order to construct the relay switching law in the form

$$
u(t) \in u_{r}(x(t))=\underset{v \in \mathcal{U}}{\operatorname{argmin}} \Gamma^{T}(t, x(t)) v,
$$

where $\Gamma: \mathbb{R}^{n+1} \rightarrow \mathbb{R}^{m}$ is a continuous (outside the origin) nonlinear function to be defined. The inclusion in (4) indicates that argmin is not unique in general case. In particular, if $m=1$ and $\mathcal{U}=\{-1,1\}$ then $u_{r}(x)=$ $-\overline{\operatorname{sign}}[\Gamma(x)]$ similarly to the sliding mode control [4], where

$$
\overline{\operatorname{sign}}[\rho]=\left\{\begin{array}{ccc}
1 & \text { if } & \rho>0 \\
-1 & \text { if } & \rho<0 \\
\{-1,1\} & \text { if } & \rho=0
\end{array}\right.
$$

Note that in order to define the control input at the current state $x(t)$ according to the formula (4) we just need to find the minimum of $\Gamma^{T}(x(t)) v$ over finite set of values $v \in \mathcal{U}$. This operation does not need applying any finite or infinite dimensional optimization procedure. We just need to calculate $N$ scalar products $\Gamma^{T}(x(t)) v_{i}, i=1, \ldots, N$ and select the minimum.

\section{PRELIMINARIES}

\section{A. Finite-Time Stability}

Let us consider the system of the form

$$
\dot{x}(t)=f(x(t)), \quad x(0)=x_{0}, \quad t \in \mathbb{R}_{+},
$$

where $x \in \mathbb{R}^{n}$ is the state vector, $f: \mathbb{R}^{n} \rightarrow \mathbb{R}^{n}$ is a nonlinear discontinuous (but locally measurable) vector field. Let Filippov procedure be applied for regularization of the discontinuous ODE, i.e by definition, an absolute continuous function $x\left(\cdot, x_{0}\right)$ is a solution to the Cauchy problem associated to (5) if $x\left(0, x_{0}\right)=x_{0}$ and almost everywhere it satisfies the differential inclusion

$$
\dot{x} \in F(x)=\bigcap_{\delta>0} \bigcap_{\mu(N)=0} \operatorname{cof}(x+B(\delta) \backslash N),
$$

where $\mu(N)=0$ means that a set $N \subset \mathbb{R}^{n}$ has measure 0 .

Let the origin be an equilibrium point of the system (5), i.e. $0 \in F(0)$. Only strong uniform stability properties of the system (5) are studied in the this paper, so the corresponding words "strong uniform" will be omitted below for shortness and simplicity of the presentation.

Definition 1 ([23], [24], [5]): The origin of system (5) is said to be finite-time stable if it is asymptotically stable and finite-time attractive, i.e. for any $x_{0} \in \mathcal{M} \backslash\{0\}$ there exists $T\left(x_{0}\right) \in \mathbb{R}_{+}$such that $x\left(t, x_{0}\right)=0$ for all $t \geq T\left(x_{0}\right)$, where $\mathcal{M}$ is a neighborhood of the origin and $T$ is called the settling-time function of the system (5). If $\mathcal{M}=\mathbb{R}^{n}$ then the origin is globally finite-time stable.

\section{B. Implicit Lyapunov Function Method}

The next theorem is utilized below in order to design the feedback law.

Theorem 2: [18] If there exists a continuous function $Q$ : $\mathbb{R}_{+} \times \mathbb{R}^{n} \rightarrow \mathbb{R}$ that satisfies the conditions

C1) $Q$ is continuously differentiable in $\mathbb{R}_{+} \times \mathbb{R}^{n} \backslash\{0\}$;

$C 2$ ) for any $x \in \mathbb{R}^{n} \backslash\{0\}$ there exist $V \in \mathbb{R}_{+}$such that $Q(V, x)=0$

$C 3)$ let $\Omega=\left\{(V, x) \in \mathbb{R}_{+} \times \mathbb{R}^{n}: Q(V, x)=0\right\}$ and

$$
\lim _{\substack{x \rightarrow 0 \\(V, x) \in \Omega}} V=0^{+}, \lim _{\substack{V \rightarrow 0^{+} \\(V, x) \in \Omega}}\|x\|=0, \lim _{\substack{\|x\| \rightarrow \infty \\(V, x) \in \Omega}} V=+\infty ;
$$

$C 4)$ the inequality $\frac{\partial Q(V, x)}{\partial V}<0$ holds for all $V \in \mathbb{R}_{+}$and $x \in \mathbb{R}^{n} \backslash\{0\}$;

C5) there exist $c \in \mathbb{R}_{+}$and $\mu \in(0,1]$ such that

$$
\sup _{t \in \mathbb{R}_{+}, y \in K[f](t, x)} \frac{\partial Q(V, x)}{\partial x} y \leq c V^{1-\mu} \frac{\partial Q(V, x)}{\partial V},(V, x) \in \Omega ;
$$

then the origin of system (5) is globally finite time stable with the following settling time estimate: $T\left(x_{0}\right) \leq \frac{V_{0}^{\mu}}{c \mu}$, where $V_{0} \in \mathbb{R}_{+}: Q\left(V_{0}, x_{0}\right)=0$.

Theorem 2 provides the sufficient conditions of finite-time stability for implicit definition of Lyapunov function. The conditions $(1)-C 4$ ) guarantee existence and uniqueness of a continuously differentiable (outside the origin) positive definite radially unbounded function $V: \mathbb{R}^{n} \rightarrow \mathbb{R}_{+}$, which is implicitly defined by the equation $Q(V, x)=0$. The implicit function theorem [28] gives

$$
\frac{\partial V}{\partial x}=-\left[\frac{\partial Q}{\partial V}\right]^{-1} \frac{\partial Q}{\partial x} .
$$

Due to conditions $C 4$ ), C5) the estimate

$$
\dot{V}(x) \leq \sup _{y \in F(x)} \frac{\partial V}{\partial x} y \leq-c V^{1-\mu}
$$

implies the finite-time stability of the origin of (5).

Corollary 3: If the conditions $C 1$ )-C4) of Theorem 2 are fulfilled then the set

$$
\varepsilon\left(V_{0}\right)=\left\{z \in \mathbb{R}^{n}: Q\left(V_{0}, z\right) \leq 0\right\}
$$

is the $V_{0}$-level set $\left\{s \in \mathbb{R}^{n}: V(s) \leq V_{0}\right\}$ of the positive definite function $V: \mathbb{R}^{n} \rightarrow \mathbb{R}_{+}$implicitly defined by the equation $Q(V, s)=0$.

Proof: Indeed, if $\tilde{s} \in \mathbb{R}^{n}$ is such that $V(\tilde{s})=\alpha$, where $\alpha \in \mathbb{R}_{+}$then $Q(\alpha, \tilde{s})=0$, i.e. $\tilde{s} \in \varepsilon(\alpha, P)$. The condition C4) implies that $Q\left(V^{\prime}, \tilde{s}\right)<0$ (i.e $\tilde{s} \in \varepsilon\left(V^{\prime}\right)$ ) for any $V^{\prime}>\alpha$ and $Q\left(V^{\prime \prime}, \tilde{s}\right)>0$ (i.e. $\tilde{s} \notin \varepsilon\left(V^{\prime}\right)$ ) for any $V^{\prime \prime}<\alpha$.

Corollary 3 allows us to adapt Theorem 2 to local finitetime stability analysis possessing the condition $C 5)$ locally, i.e. $0<V<\bar{V}$ and $x \in \varepsilon(\bar{V})$ for some given $\bar{V} \in \mathbb{R}_{+}$. In this case the level set $\varepsilon(\bar{V})$ specifies the finite-time attraction domain $\mathcal{M}$ (see Definition 1). 


\section{IMPLICIT RELAY FEEDBACK LAW}

\section{A. Block Decomposition}

Let us initially decompose the original multi-input system (1) to a block form [25]. The block decomposition procedure studied in [26], [22] is briefly discussed in Appendix. It constructs the non-singular coordinate transformation

$$
s=\Theta x
$$

reducing the original system (1) to the block form

$$
\dot{s}(t)=\tilde{A} s+\tilde{B}\left(u+K_{\text {lin }} s\right),
$$

where $K_{\text {lin }} \in \mathbb{R}^{m \times n}$ is a rectangular matrix,

$$
\tilde{A}=\left(\begin{array}{cccc}
0 & A_{12} & \ldots & 0 \\
\ldots & \ldots & \ldots & \ldots \\
0 & \ldots & \ldots & A_{k-1 k} \\
0 & \ldots & \ldots & 0
\end{array}\right), \tilde{B}=\left(\begin{array}{c}
0 \\
\ldots \\
0 \\
A_{k k+1}
\end{array}\right) \in \mathbb{R}^{n \times n_{k}},
$$

$A_{i+1} \in \mathbb{R}^{n_{i} \times n_{i+1}}$ are matrices of full row rank, $i=$ $1,2, . ., k, n_{1}+\ldots+n_{k}=n, n_{k}=m$ and $B_{0} \in \mathbb{R}^{m \times m}$ is a nonsingular matrix.

\section{B. Relay Feedback Design}

Introduce the ILF function

$$
Q(V, s):=s^{T} D_{r}\left(V^{-1}\right) P D_{r}\left(V^{-1}\right) s-1,
$$

where $s=\left(s_{1}, \ldots, s_{k}\right)^{T}, s_{i} \in \mathbb{R}^{n_{i}}, V \in \mathbb{R}^{+}, D_{r}(\lambda)$ is the dilation matrix of the form

$$
D_{r}(\lambda)=\left(\begin{array}{cccc}
\lambda^{r_{1}} I_{n_{1}} & 0 & \cdots & 0 \\
0 & \lambda^{r_{2}} I_{n_{2}} & \cdots & 0 \\
\ldots & \cdots & \cdots & \cdots \\
0 & \cdots & 0 & \lambda^{r_{k}} I_{n_{k}}
\end{array}\right)
$$

with

$\lambda \in \mathbb{R}_{+}, \quad r_{i}=1+(k-i) \mu, \quad i=1,2, . ., k, \quad 0<\mu \leq 1$ and $P \in \mathbb{R}^{n \times n}$ is a symmetric positive definite matrix, i.e. $P=P^{T}>0$. Denote $H_{\mu}:=\operatorname{diag}\left\{r_{i} I_{n_{i}}\right\}_{i=1}^{k}-$ the block diagonal matrix.

Theorem 4: Let $\mu \in(0,1), X \in \mathbb{R}^{n \times n}$ and $Y \in \mathbb{R}^{n_{k} \times n}$ satisfy the system of matrix inequalities:

$$
\begin{gathered}
\tilde{A} X+X \tilde{A}^{T}+\tilde{B} Y+Y^{T} \tilde{B}^{T}+H_{\mu} X+X H_{\mu}=0, \\
X H_{\mu}+H_{\mu} X>0, \quad X>0 .
\end{gathered}
$$

Consider $V: \mathbb{R}^{n} \rightarrow \mathbb{R}_{+}$implicitly defined by the equation $Q(V, s)=0$ and the function $Q$ given by (11) with $P:=$ $X^{-1}$. Then the control of the form (4) with

$$
\Gamma^{T}(x)=x^{T} \Theta^{T} D_{r}\left(\frac{1}{V(\Theta x)}\right) P D_{r}\left(\frac{1}{V(\Theta x)}\right) \tilde{B},
$$

locally stabilizes the origin of the system (1) in a finite time. The settling-time function is bounded as follows

$$
T\left(x_{0}\right) \leq \frac{V_{0}^{\mu}}{\mu}, \quad \forall x_{0} \in \varepsilon(\bar{V})
$$

where $V_{0} \in \mathbb{R}_{+}: Q\left(V_{0}, \Theta x_{0}\right)=0$, the set $\varepsilon(\bar{V})$ is the finite-time attraction given by (7) with a positive $\bar{V} \in \mathbb{R}_{+}$:

$$
\bar{V}=\sup _{V \in \mathbb{R}_{+}: \varepsilon(V) \subset \mathcal{C}_{\mathcal{U}}} V
$$

$\mathcal{C}_{\mathcal{U}}=\left\{z \in \mathbb{R}^{n}: V^{1-\mu}(\Theta z) Y P^{-1} D_{r}\left(V^{-1}(\Theta z)\right) \Theta z \in \operatorname{co}(\mathcal{U})\right\}$

The system of matrix inequalities (13) can be easily solved using LMI toolbox of MATLAB or, for example, SeDuMi solver. The solution of (13) also can be constructed analytically using the proof (see, [22]) of the next proposition.

Proposition 5 ([22]): The system of matrix inequalities (13) is feasible for any $\mu \in \mathbb{R}_{+}$.

\section{PRACTICAL IMPLEMENTATION}

In order to realize the control algorithm (4), (14) in practice we need to know $V$. In some cases the function $V$ can be calculated analytically. See, for example [27], where the analytical derivation has been provided for the case $n=2, m=1$. The function $V$ can also be approximated numerically on a grid, which is constructed in the finitetime attraction domain $\varepsilon(\bar{V})$. Finally, the relay control law (4) can be applied by means of on-line estimation of $V$. The following corollary may be utilized for this purpose.

Corollary 6: If

1) the conditions of Theorem 4 hold;

2) $\left\{t_{i}\right\}_{i=0}^{+\infty}$ is an arbitrary sequence of time instances such that $0=t_{0}<t_{1}<t_{2}<\ldots$ and $\lim _{i \rightarrow+\infty} t_{i}=+\infty$;

3 ) the relay control $u_{r}$ has the form (4) with the sampled computation of the switching function $\Gamma(t, x)=\tilde{\Gamma}_{i}(\Theta x)$ for $t \in\left[t_{i}, t_{i+1}\right)$, where

$$
\tilde{\Gamma}_{i}^{T}(s)=s^{T} D_{r}\left(V_{i}^{-1}\right) P D_{r}\left(V_{i}^{-1}\right) \tilde{B}, \quad Q\left(V_{i}, s\left(t_{i}\right)\right)=0 .
$$

Then the closed-loop system (1), (4) is globally asymptotically stable.

The corollary shows that if a the switching function is re-computed only at a countable number of time instants $t_{i}$, then the asymptotic stability of the closed loop system is guaranteed. In practice, the estimation of the switching parameter $V_{i}$ can be obtained using the following algorithm [22].

$$
\begin{aligned}
& \text { Algorithm 7: } \\
& \text { INITIALIZATION: } V_{0}=1 ; a=V_{\min } ; \quad b=1 ; \\
& \text { STEP : } \\
& \text { If } s_{i}^{T} D_{r}\left(b^{-1}\right) P D_{r}\left(b^{-1}\right) s_{i}>1 \text { then } a=b ; b=2 b ; \\
& \text { elseif } s_{i}^{T} D_{r}\left(a^{-1}\right) P D_{r}\left(a^{-1}\right) s_{i}<1 \text { then } \\
& \quad b=a ; a=\max \left\{\frac{a}{2}, V_{\min }\right\} ; \\
& \text { else } \\
& \quad c=\frac{a+b}{2} ; \\
& \quad \text { If } s_{i}^{T} D_{r}\left(c^{-1}\right) P D_{r}\left(c^{-1}\right) s_{i}<1 \text { then } b=c ; \\
& \quad \text { else } a=\max \left\{V_{\min }, c\right\} ; \\
& \text { endif; } \\
& V_{i}=b ;
\end{aligned}
$$

If $s_{i} \in \mathbb{R}^{n}$ is some given vector and STEP of the presented algorithm is applied recurrently many times to the same $s_{i}$ then Algorithm 7 realizes:

1) a localization of the unique positive root of the equation $Q\left(V, s_{i}\right)=0$, i.e. $V_{i} \in[a, b]$;

2) improvement of the obtained localization by means of the bisection method, i.e. $(b-a) \rightarrow 0$. 
Such an application of Algorithm 7 allows us to calculate $V_{i}$ with rather high precision but it requests a high computational capability of a control device. If the computational power is very restricted, then STEP of Algorithm 7 may be realized just once at each sampled instant of time. Indeed, in the proof of Corollary 6 we show that the ellipsoid $\varepsilon\left(V_{i}\right)$ is an invariant set of the closed-loop system (1), (4) with $\Gamma(x)=\tilde{\Gamma}_{i}(\Theta x)$. If the root of the equation $Q\left(V, s_{i}\right)=0$ is localized, Algorithm 7 always selects the upper estimate of $V_{i}$ providing that $s\left(t_{i}\right) \in \varepsilon\left(V_{i}\right)$, i.e. $V_{i}$ do not increase in time.

The parameter $V_{\min }$ defines lower admissible value of $V$. In practice, this parameter cannot be selected arbitrary small due to finite numerical precision of digital devices and measurement errors, which may imply $s\left(t_{i}\right) \notin \varepsilon\left(V_{i}\right)$. Therefore, the real-life realization of the relay control may provide the only practical stabilization of the system with the attractive set $\varepsilon\left(V_{\min }\right)$.

\section{ACADEMIC EXAMPLE}

Let us consider the system (1) with

$$
A=\left(\begin{array}{ccc}
0 & 1 & -0.5 \\
1 & -0.3 & 0.9 \\
0.5 & 0 & 0.7
\end{array}\right), \quad B=\left(\begin{array}{cc}
0 & 0 \\
0.5 & 0 \\
0 & 1
\end{array}\right)
$$

and $\mathcal{U}=\left\{v_{1}, v_{2}, v_{3}\right\}$,

$$
v_{1}=\left(\begin{array}{c}
0 \\
\frac{\sqrt{3}}{3}
\end{array}\right), v_{2}=\left(\begin{array}{c}
0.5 \\
-\frac{\sqrt{3}}{6}
\end{array}\right), v_{3}=\left(\begin{array}{c}
-0.5 \\
-\frac{\sqrt{3}}{6}
\end{array}\right) .
$$

The considered system already has the required block form (9) with $\tilde{B}=B$

$$
\tilde{A}=\left(\begin{array}{ccc}
0 & 1 & -0.5 \\
0 & 0 & 0 \\
0 & 0 & 0
\end{array}\right), K_{l i n}=\left(\begin{array}{ccc}
2 & -0.6 & 1.8 \\
0.5 & 0 & 0.7
\end{array}\right) .
$$

The solution to the LMI (13) was obtained for $\mu=0.999$ :

$$
\begin{gathered}
X=\left(\begin{array}{ccc}
0.0109 & -0.0109 & 0.0218 \\
-0.0109 & 0.0374 & 0.0206 \\
0.0218 & 0.0206 & 0.1496
\end{array}\right), \\
Y=\left(\begin{array}{ccc}
0.0114 & -0.0748 & -0.0412 \\
-0.0112 & -0.0207 & -0.1496
\end{array}\right) .
\end{gathered}
$$

The relay control (4) with $\Gamma$ of the form (14), $P=X^{-1}$ is applied using the algorithm 7 with $V_{\min }=10^{-3}$. The explicit Euler discretization with the step size $10^{-3}$ is utilized for the numerical simulation. The trajectories are depicted on Fig. 1.

The upper estimate of the settling time (15) gives 1.4734 . The simulation confirms this.

The numerical simulation (see Fig. 2) shows fast control switchings approving the expectable fact that the finitetime stabilization by means of the relay control implies appearance of sliding mode in the closed-loop system.

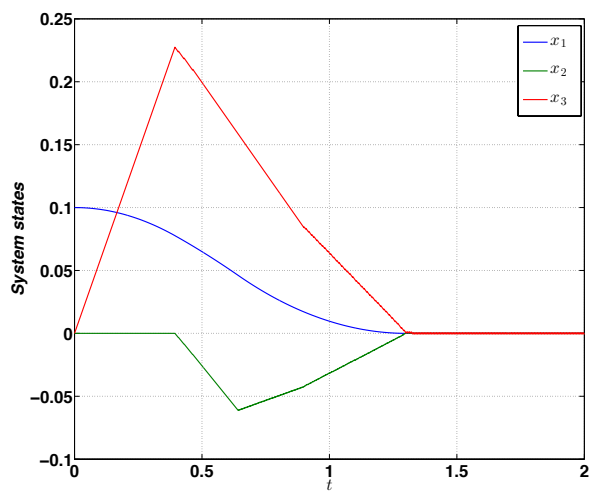

Fig. 1. Evolution of the system states of the closed-loop system

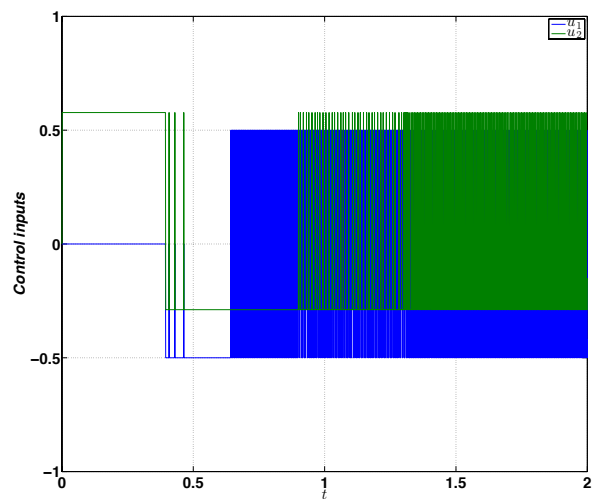

Fig. 2. Control signals

\section{CONCLUSION}

The paper presents relay feedback control algorithm for stabilization of linear multi-input system provided nonasymptotic transitions. The control design procedure combines the ILF method and convex embedding technique. This approach allows us to provide simple procedure for implicit switching surface design using LMIs. The algorithm of practical implementation of the obtained implicit relay feedback is also presented and justified. The robustness analysis of the proposed control scheme is considered as the subject for future research.

\section{REFERENCES}

[1] I. Flugge-Lotz, Discontinuous automatic control. Princeton University Press, 1953.

[2] Y. Z. Tsypkin, Relay Control Systems. Moscow: Nauka, 1984.

[3] I. Boiko, Discontinuous Control Systems: Frequency-Domain Analysis and Design. Springer, 2009.

[4] V. Utkin, J. Guldner, and J. Shi, Sliding Mode Control in ElectroMechanical Systems. $\quad$ CRC Press., 2009.

[5] Y. Orlov, "Finite time stability and robust control synthesis of uncertain switched systems," SIAM Journal of Control and Optimization, vol. 43(4), pp. 1253-1271, 2005.

[6] A. Levant, "Principles of 2-sliding mode design," Automatica, vol. 43, pp. 576-586, 2007.

[7] A. Polyakov and A. Poznyak, "Unified Lyapunov function for a finitetime stability analysis of relay second-order sliding mode control systems," IMA Journal of Mathematical Control and Information, vol. 29(4), pp. 529-550, 2012.

[8] G. Bartolini, A. Pisano, E. Punta, and E. Usai, "A survey of applications of second-order sliding mode control to mechanical systems," International Journal of Control, vol. 76(9/10), pp. 875-892, 2003. 
[9] A. Van Der Schaft and H. Schumacher, An introduction to hybrid dynamical systems. Springer, 2000.

[10] D. Liberzon, Switching in Systems and Control. Birkhauser, 2003.

[11] R. Shorten, F. Wirth, O. Mason, K. Wulff, and C. King, "Stability criteria for switched and hybrid systems," Invited paper for SIAM Review, vol. 49, no. 4, pp. 545-592, 2007.

[12] P. Bolzern and W. Spinelli, "Quadratic stabilization of a switched affine system about a nonequilibrium point," in Proceeding of the 2004 American Control Conference, Boston, Massachusetts, USA, 2004.

[13] G. Deaecto, J. Geromel, F. Garcia, and J. Pomilio, "Switched affine systems control design with application to dc-dc converters," Control Theory Applications, IET, vol. 4, no. 7, pp. 1201 -1210, july 2010.

[14] X. Xu and G. Zhai, "Practical stability and stabilization of hybrid and switched systems," IEEE Transactions on Automatic Control, vol. 50, no. 11, pp. 1897-1903, 2005.

[15] L. Hetel, E. Fridman, and T. Floquet, "Variable structure control with generalized relays: A simple convex optimization approach," IEEE Transactions on Automatic Control, vol. 60(2), pp. 497-502, 2015.

[16] V. Korobov, "A solution of the problem of synthesis using a controllability function," Doklady Academii Nauk SSSR, vol. 248, pp. 10511063, 1979.

[17] J. Adamy and A. Flemming, "Soft variable-structure controls: a survey," Automatica, vol. 40, pp. 1821-1844, 2004.

[18] A. Polyakov, D. Efimov, and W. Perruquetti, "Finite-time and fixedtime stabilization: Implicit lyapunov function approach," Automatica, vol. 51, pp. 332-340, 2015.

[19] G. Bartolini, E. Punta, and T. Zolezzi, "Simplex sliding mode control of multi-input systems with chattering reduction and mono-directional actuators," Automatica, vol. 47, pp. 2433-2437, 2011.

[20] S. V. Bajda and D. B. Izosimov, "Vector method of design of sliding motion and simplex algorithms," Automation and Remote Control, vol. 46, pp. 830-837, 1985 .

[21] A. Filippov, Differential equations with discontinuous right-hand sides. Kluwer, Dordrecht, 1988.

[22] A. Polyakov, D. Efimov, and W. Perruquetti, "Robust stabilization of mimo systems in finite/fixed time," International Journal of Robust and Nonlinear Control, 2015.

[23] E. Roxin, "On finite stability in control systems," Rendiconti del Circolo Matematico di Palermo, vol. 15(3), pp. 273-283, 1966.

[24] S. Bhat and D. Bernstein, "Finite-time stability of continuous autonomous systems," SIAM Journal of Control and Optimization, vol. 38(3), pp. 751-766, 2000

[25] S. Drakunov, D. Izosimov, A. Lukyanov, V. Utkin, and V. Utkin, "Block control principle I," Automation and Remote Control, vol. 51(5), pp. 601-609, 1990

[26] A. Polyakov, "Nonlinear feedback design for fixed-time stabilization of linear control systems," IEEE Transactions on Automatic Control, vol. 57(8), pp. 2106-2110, 2012.

[27] A. Polyakov and I. Chairez, "A new homogeneous quasi-continuous second order sliding mode control," in Latinamerican Congress on Automatic Control, Cancun, Mexico, 2014.

[28] R. Courant and F. John, Introduction to calculus and analysis (Vol. II/1). New York: Springer, 2000.

\section{APPENDIX}

\section{A. Transformation to Block Form}

Let us denote by rown $(W)$ the number of rows of a matrix $W$ and by $\operatorname{null}(W)$ the matrix that has the columns defining an orthonormal basis of the null space of a matrix $W$.

Let the orthogonal matrices $T_{i}$ be defined by the following simple algorithm:

Initialization : $A_{0}=A, B_{0}=B, T_{0}=I_{n}, k=0$.

Loop: While $\operatorname{rank}\left(B_{k}\right)<\operatorname{rown}\left(A_{k}\right) \quad$ do

$$
\begin{gathered}
A_{k+1}=B_{k}^{\perp} A_{k}\left(B_{k}^{\perp}\right)^{T}, \quad B_{k+1}=B_{k}^{\perp} A_{k} \hat{B}_{k}, \\
T_{k+1}=\left(\begin{array}{c}
B_{k}^{\perp} \\
\hat{B}_{k}
\end{array}\right), \quad k=k+1,
\end{gathered}
$$

where $B_{k}^{\perp}=\left(\operatorname{null}\left(B_{k}^{T}\right)\right)^{T}, \hat{B}_{k}=\left(\operatorname{null}\left(B_{k}^{\perp}\right)\right)^{T}$.
In the paper [26] it was proven that the orthogonal matrix

$$
\begin{gathered}
G=\left(\begin{array}{cc}
T_{k} & 0 \\
0 & I_{w_{k}}
\end{array}\right)\left(\begin{array}{cc}
T_{k-1} & 0 \\
0 & I_{w_{k-1}}
\end{array}\right) \ldots\left(\begin{array}{cc}
T_{2} & 0 \\
0 & I_{w_{2}}
\end{array}\right) T_{1}, \\
\text { where } w_{i}:=n-\operatorname{rown}\left(T_{i}\right)
\end{gathered}
$$

provides

$$
\begin{gathered}
G A G^{T}=\left(\begin{array}{ccccc}
A_{11} & A_{12} & 0 & \ldots & 0 \\
A_{21} & A_{22} & A_{23} & \ldots & 0 \\
\ldots & \ldots & \ldots & \ldots & \ldots \\
A_{k-11} & A_{k-12} & \ldots & A_{k-1 k-1} & A_{k-1 k} \\
A_{k 1} & A_{k 2} & \ldots & A_{k k-1} & A_{k k}
\end{array}\right), \\
G B=\left(\begin{array}{llllll}
0 & 0 & \ldots & 0 & A_{k k+1}^{T}
\end{array}\right)^{T},
\end{gathered}
$$

where $A_{k k+1}=\hat{B}_{0} B_{0}, A_{i j} \in \mathbb{R}^{n_{i} \times n_{j}}, n_{i}:=\operatorname{rank}\left(B_{k-i}\right)$, $i, j=1,2, \ldots, k$ and $\operatorname{rank}\left(A_{i+1}\right)=n_{i}$.

Recall that the $B$ has full column $\operatorname{rank}(\operatorname{rank}(B)=$ $m)$. Consequently, $A_{k k+1}$ is square and nonsingular. Since $\operatorname{rank}\left(A_{i i+1}\right)=n_{i}=\operatorname{rown}\left(A_{i+1}\right)$ then $A_{i+1} A_{i+1}^{T}$ is invertible and $A_{i+1}^{+}=A_{i+1}^{T}\left(A_{i+1} A_{i i+1}^{T}\right)^{-1}$ is the right inverse matrix of $A_{i i+1}$. Introduce the linear coordinate transformation $s=\Phi y, s=\left(s_{1}, \ldots, s_{k}\right)^{T}, s_{i} \in \mathbb{R}^{n_{i}}$, $y=\left(y_{1}, \ldots, y_{k}\right)^{T}, y_{i} \in \mathbb{R}^{n_{i}}$ by the formulas:

$$
\begin{gathered}
s_{i}=y_{i}+\varphi_{i}, \quad i=1,2, \ldots, k, \quad \varphi_{1}=0, \\
\varphi_{i+1}=A_{i i+1}^{+}\left(\sum_{j=1}^{i} A_{i j} y_{j}+\sum_{r=1}^{i} \frac{\partial \varphi_{i}}{\partial y_{r}} \sum_{j=1}^{r+1} A_{r j} y_{j}\right) .
\end{gathered}
$$

The presented coordinate transformation is linear and nonsingular. The inverse transformation $y=\Phi^{-1} s$ is defined as follows:

$$
\begin{gathered}
y_{i}=s_{i}+\psi_{i}, \quad i=1,2, \ldots, k, \quad \psi_{1}=0 \\
\psi_{i+1}=A_{i i+1}^{+}\left(\sum_{k=1}^{i} \frac{\partial \psi_{i}}{\partial s_{k}} A_{i i+1} s_{k+1}-\sum_{j=1}^{i} A_{i j}\left(s_{j}+\psi_{j}\right)\right) .
\end{gathered}
$$

For example, if $k=3$ then the matrix $\Phi$ has the form

$$
\Phi=\left(\begin{array}{ccc}
I_{n_{1}} & 0 & 0 \\
A_{12}^{+} A_{11} & I_{n_{2}} & 0 \\
A_{23}^{+}\left(A_{21}+A_{12}^{+} A_{11}^{2}\right) & A_{23}^{+}\left(A_{22}+A_{12}^{+} A_{11} A_{12}\right) & I_{n_{3}}
\end{array}\right) .
$$

In general case, the transformation $\Phi$ can be calculated numerically.

Applying the transformation $s=\Theta x$ with $\Theta=\Phi G$ to the system (1) we obtain the system

$$
\dot{s}=\left(\begin{array}{cccc}
0 & A_{12} & \ldots & 0 \\
\ldots & \ldots & \ldots & \ldots \\
0 & \ldots & \ldots & A_{k-1 k} \\
\tilde{A}_{k 1} & \ldots & \ldots & \tilde{A}_{k k}
\end{array}\right) s+\tilde{B} u,
$$

which is equivalent to (9) with

$$
K_{l i n}=B_{0}^{+}\left(\begin{array}{llll}
\tilde{A}_{k 1} & \ldots & \ldots & \tilde{A}_{k k}
\end{array}\right)
$$

(please see [26] for more details). 